Contents List available at RAZI Publishing

Matriks Sains Matematik (MSMK)

urnal Homepage: http://www.razipublishing.com/journals/matriks-sainsmatematik

https://doi.org/10.26480/msmk.01.2017.34.39

\title{
Jackknife Replication Variance Estimation of Population Total Under Systematic Sampling With Varying Probabilities
}

\author{
Khadija Fatima1 $^{*}$, Zahoor Ahmed ${ }^{1}$ and Abeeda Fatima ${ }^{1}$ \\ ${ }^{1}$ Department of Statistics, University of Gujrat, Gujrat, Pakistan*Corresponding Author email: khadija.fatima@uog.edu.pk
}

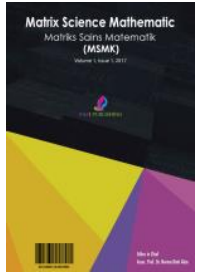

This is an open access article distributed under the Creative Commons Attribution License, which permits unrestricted use, distribution, and reproduction in any medium, provided the original work is properly cited

\section{ARTICLE DETAILS}

Article history:

Received 16 July 2016

Accepted 15 November 2016

Available online 12 January 2017

Keywords:

Multi-Auxiliary Variables, Horvitz and Thompson Estimator, Madow Sampling Procedure, Jackknife Replication Variance Estimation

\section{ABSTRACT}

Systematic sampling with probability proportional to size either randomized or non-randomized, has been used in a number of large scale household survey. Even this sampling technique has been widely used in forestry for the estimation of volume of timber. So in this paper general class of estimators has been developed for estimating population total using multi-auxiliary variables under Horvitz and Thompson estimator for systematic sampling. The expressions of bias and mean square error derived up to first order approximation along with the Jackknife replication variance estimation. The detailed simulation study has also been carried using madow systematic sampling procedure.

\section{INTRODUCTION}

The generic term used for sampling design is the rule that direct the sample selection. The sampling procedure includes two important general ideas: inclusion probabilities and design weights. The inclusion probability of a unit is identified as the probability with which it is selected under sampling design. The design weight of an element is calculated as the inverse of its inclusion probability, which expected to be greater than zero for all units (Sarndal and Lundstrom, 2005).

The use of the systematic sampling technique involves the assumption that it is possible to order the units of population in a logical way, and then use this ordering in selecting the sample systematically (Madow and Madow, 1944). Systematic sampling with probability proportional to size either randomized or non-randomized, has been used in a number of large scale household survey. Scientest used non randomized (PPS) systematic sampling in the Indian National Sample Survey (Murthy, 1967). Some of researcher also used (PPS) systematic sampling in survey on employment in Greece for choosing units from each stratum (Raj, 1967). The theoretical basis for systematic sampling for stratified and unstratified population proposed (Madow and Madow, 1944). Recently, scienctist introduced the procedure of sampling with unequal probability and without replacement (Madow, 1949). The concept of unequal probability sampling without replacement was first given (Horvitz and Thompson, 1952).

Beside that, the researcher also provided the variance formula for the population total derived $O\left(N^{-4}\right)$ an approximation of pair wise inclusion

probabilities $\pi_{i i i^{\prime}}$ under randomized systematic PPS sampling (Hartley and Rao, 1962). The variance expression under HT estimator of population total say $\hat{Y}=\sum_{i=1}^{n} \frac{y_{i}}{\pi}$ and retaining only $O(N)$ and they obtained

$$
V(\hat{Y})=\sum_{i=1}^{N} \pi_{i}\left[1-\frac{(n-1)}{n} \pi_{i}\right]\left(\frac{y_{i}}{\pi_{i}}-\frac{Y}{n}\right)^{2}
$$

There are some or the researcher which introduced a weighted Jackknife procedure (Roy and Safiquzzaman, 2002). They worked on Jackknife variance estimation for single-phase sampling attached a weight function $\boldsymbol{w}_{i}$ to each pseudo-value $t_{i}$, and defined the weighted Jackknife estimators of the population total as;

$$
t_{j k}(w)=\frac{\sum_{s} \frac{w_{i}}{\pi_{i}} t_{i}}{\sum_{s} \frac{w_{i}}{\pi_{i}}} \text { Where } w_{i} \geq 0 \text { and } \sum_{U} w_{i}=1
$$

There are several methods of replication variance estimation, but here we discussed about the jackknife method of variance estimation. The Jackknife variance estimation is suitable for many models and survey designs. There are concept of the Jackknife was first introduced for estimating the bias for nonlinear estimation (Quenouille, 1949). This technique used for the purpose of variance estimation (Tukey, 1958). Some of researcher also provided a rigorous investigation of the replication method and considered replication for different types of estimators (Kim et al., 2006). Beside, some of them also have discussed the replication variance estimation under two-phase sampling (Kim and Yu, 2011).

The main objective of this paper is to develop generalized regression cum ratio estimators for population total using multi auxiliary variables along with its Jacknife variance estimates for systematic sampling with varying probabilities. The expressions for 
bias and mean square error (MSE) for the proposed class of estimators derived up to $1^{\text {st }}$ order approximation. The confidence intervals of population totals are also provided for proposed estimators.

\section{SYSTEMATIC SAMPLING DESIGN WITH VARYING PROBABILITY}

Most of the researcher choosed systematic sampling for its simplicity and its periodic quality. The unequal probability of systematic sample are sometimes called systematic PPS sampling. In the very first step all the units in the population are arranged at random in a list; can be sited in a specific order or may be presented in naturally happen. In the present study the sample selection procedure is then used by systematic method, when the order of the population units is random (Madow, 1949).

Then in Second, select a random start i.e. select a uniform variable $d$ with $0 \leq \mathrm{d}<1$. Then the $n$ selected units are those whose index satisfied this $\mathrm{II}_{\mathrm{j}-1} \leq d+k<\mathrm{II}_{\mathrm{j}}$ for some $k=0$ and $n-1$. Since ${ }_{n p_{i}} \leq 1$ every one of the $\mathrm{n}$ integers $k=0,1, \ldots, n-1$ will select a different sampling unit $j$. The joint inclusion probabilities for unit $i$ and $j$ only depend on the first order inclusion probabilities.

\subsection{Regression Cum Ratio Estimator}

A scientist who first time given the concept of combining regression and ratio methods and named it a regression-cum-ratio estimator when we have at least two auxiliary variable (Mohanty, 1967). This approach is appealing because if we have multiple auxiliary variables then it is not necessary that all have same relation with the outcome variable i.e. either the regression line passes through the origin or has some intercept. Consider $N$ units $u_{1}, u_{2}, \ldots, u_{N}$ from finite population. Let $Y$ be a quantitative character, taking the values of $y_{k}$ on $u_{k},(1 \leq k \leq N)$. Let $n$ be a sample size of units selected from $N$ using systematic PPS sampling without replacement when population is in random order. Furthermore, let $X_{1}, X_{2}, \ldots, X_{q}$ are $q$ auxiliary variables and $X_{i}$ denote the known population total of $i^{\text {th }}$ auxiliary variable and $\mathrm{Y}$ is a population total for study variable.

The research also provided the variance formula for population total derived $O\left(N^{-4}\right)$ an approximation of pairwise inclusion probabilities $\pi_{k k^{\prime}}$ under randomized systematic PPS sampling (Hartley and Rao, 1962). The variance expression for HT estimator of population total say $\sum_{t_{y}^{\prime}=\sum_{k=1}^{n} \frac{y_{k}}{\pi_{k}}}$ and

retaining only $O(N)$ and they obtained

$V\left(t_{y}^{\prime}\right)=\sum_{k=1}^{N} \pi_{k}\left[1-\frac{(n-1)}{n} \pi_{k}\right]\left(\frac{y_{k}}{\pi_{k}}-\frac{Y}{n}\right)^{2}$.

where $\mathbf{e}_{x_{r}}, \mathbf{e}_{x_{s}}$ and $\mathbf{e}_{v}$ are the sampling errors that are expected to be very small and assumed that $E\left(\mathbf{e}_{x_{r}}\right)=E\left(\mathbf{e}_{x_{s}}\right)=E\left(\mathbf{e}_{y}\right)=0$ and also

$E\left(\mathbf{e}_{x}^{2}\right)=\left[\sum_{k=1}^{N} \Delta_{k} d_{x_{i}}^{2}\right]_{q \times 1}=\Delta_{x}$

where

$$
\Delta_{k}=\pi_{k}-\frac{n-1}{n} \pi_{k} \pi_{k^{\prime}}
$$

$d_{y}^{2}=\left(\frac{y_{k}}{\pi_{k}}-\frac{Y}{n}\right)^{2}, d_{x}^{2}=\left(\frac{x_{k}}{\pi_{k}}-\frac{X}{n}\right)^{2}$ and

$d_{x} d_{y}=\left(\frac{x_{k}}{\pi_{k}}-\frac{X}{n}\right)\left(\frac{y_{k}}{\pi_{k}}-\frac{Y}{n}\right)$,

$d_{x} d_{x}=\left(\frac{x_{k}}{\pi_{k}}-\frac{X}{n}\right)\left(\frac{x_{k^{\prime}}}{\pi_{k^{\prime}}}-\frac{X}{n}\right)$ where $X$ and $Y$ is population total.

The general class of estimators by combining the regression approach with ratio as follows

$$
t_{g m_{n r}}=\left[t_{y}^{\prime}+a \sum_{i=1}^{r} \alpha_{i}\left(t_{x_{i}}^{\prime}-X_{i}\right)\right]\left[\prod_{i=r+1}^{s}\left(\frac{X_{i}}{t_{x_{i}}^{\prime}}\right)^{c \gamma_{i}}\right]
$$

where $a$ and $c$ are appropriate constants to be chosen for making the special cases. Let $t_{x_{i}}^{\prime}$ denote the sample total of $i^{\text {th }}$ auxiliary variable and $t_{y}^{\prime}$ be the sample total of study variable, where $t_{x_{i}}^{\prime}$ and $\boldsymbol{t}_{y}^{\prime}$ are HT estimators of total. The value of $\boldsymbol{\alpha}^{\prime} s$ and $\gamma^{\prime} s$ are determined by minimizing the MSE. As in suggested class, we divided $q$ auxiliary variables in two groups $r+s=q$; where the first $r$ variables are considered for regression estimator, second $S$ variables are considered for ratio estimator. The unknown constants $\boldsymbol{\alpha}^{\prime} S$ and $\boldsymbol{\gamma}^{\prime} S$ is getting by minimizing the MSE of $t_{g m_{r c r}}$ given in (2.1.1).

Now let $t_{y}^{\prime}=Y+e_{y}$ and $t_{x_{i}}^{\prime}=X_{i}+e_{x_{i}}$ are sampling errors of study and auxiliary variables respectively. The expression of regression cum ratio estimator becomes

$$
\begin{aligned}
& t_{g m_{r c r}}=\left[\left(Y+e_{y}\right)+a \sum_{i=1}^{r} \alpha_{i}\left(X_{i}+e_{x_{i}}-X_{i}\right)\right] \\
& {\left[\prod_{i=r+1}^{s}\left(\frac{X_{i}}{X_{i}+e_{x_{i}}}\right)^{c \gamma_{i}}\right]} \\
& t_{g m_{r c r}}=\left[Y+e_{y}+a \sum_{i=1}^{r} \alpha_{i}\left(e_{x_{i}}\right)\right]\left[\left(1-\sum_{i=r+1}^{s} \frac{c \gamma_{i}}{X_{i}} e_{x_{i}}\right)\right]
\end{aligned}
$$

Ignoring $2^{\text {nd }}$ and higher order terms and we will get

$$
\begin{aligned}
& \left(t_{g m_{r c r}}-Y\right)=e_{y}+a \sum_{i=1}^{r} \alpha_{i}\left(e_{x_{i}}\right)-Y c \sum_{i=r+1}^{s} \frac{\gamma_{i}}{X_{i}} e_{x_{i}} \\
& -c \sum_{i=r+1}^{s} \frac{\gamma_{i}}{X_{i}} e_{x_{i}} e_{y}-a c \sum_{i=1}^{r} \alpha_{i}\left(e_{x_{i}}\right) \sum_{i=r+1}^{s} \frac{\gamma_{i}}{X_{i}} e_{x_{i}}
\end{aligned}
$$

In matrix notation can be written as

$$
\begin{aligned}
& t_{g m_{r c r}}-Y=e_{y}+a \boldsymbol{\alpha}^{\prime} \mathbf{e}_{r}-c Y \boldsymbol{\gamma}^{\prime} \mathbf{X}_{s} \mathbf{e}_{s} \\
& -c \boldsymbol{\gamma}^{\prime} \mathbf{X}_{s} \mathbf{e}_{s} e_{y}-a c \boldsymbol{\alpha}^{\prime} \mathbf{e}_{r} \mathbf{e}_{s} \mathbf{X}_{s} \boldsymbol{\gamma}
\end{aligned}
$$

where $\boldsymbol{\alpha}^{\prime}$ and $\mathbf{e}_{r}$ are the vectors of unknown coefficients of order $(1 \times r)$ and sampling errors of order $(r \times 1)$ respectively for $r$ auxiliary variables. Also $\gamma^{\prime}$ and $\mathbf{e}_{s}$ are the vectors of unknown coefficients of order $(1 \times s)$ and sampling errors of order $(s \times 1)$ respectively for $S$ auxiliary variables. Let consider diagonal matrix of $S$ variables are $\mathbf{X}_{s}=\left[\frac{1}{X_{i}}\right]_{s \times s}$.

The expression of bias by taking expectation of eq (2.1.2)as

$$
\operatorname{Bias}\left(t_{g m_{r c r}}\right)=-c \boldsymbol{\gamma}^{\prime} \mathbf{X}_{s} \mathbf{S}_{y x_{s}}-a c \boldsymbol{\alpha}^{\prime} \mathbf{\Sigma}_{x_{r} x_{s}} \mathbf{X}_{s} \boldsymbol{\gamma} \text {. }
$$

where $\mathbf{e}_{r}, \mathbf{e}_{s}$ and $\mathbf{e}_{y}$ are the sampling errors that are expected to be very small quantities and assumed that $E\left(\mathbf{e}_{r}\right)=E\left(\mathbf{e}_{s}\right)=E\left(\mathbf{e}_{y}\right)=0$ and also

$$
E\left(\mathbf{e}_{r} \mathbf{e}_{s}^{\prime}\right)=\left[\sum_{k=1}^{N} \sum_{l=1}^{N} \Lambda_{k l} \hat{x}_{k i} \hat{x}_{l j}\right]_{r \times s}=\Sigma_{x_{r} x_{s}} \text { and }
$$


$E\left(\mathbf{e}_{s} \mathbf{e}_{y}\right)=\left[\sum_{k=1}^{N} \Lambda_{k l} \hat{y}_{k} \hat{x}_{k i}\right]_{s \times 1}=\mathbf{S}_{y x_{s}}$

The expression for mean square error to term order $(1 / \mathrm{n})$ from $(2.1 .2)$ fomed as:

$E\left(t_{g_{m_{r c r}}}-Y\right)^{2}=E\left(e_{y}+\boldsymbol{\alpha}^{\prime} \mathbf{e}_{r}-Y \gamma^{\prime} \mathbf{X}_{s} \mathbf{e}_{s}\right)^{2}$,

and for optimum value of $\boldsymbol{\alpha}$ and $\boldsymbol{\gamma}$, differentiating the above equation of (2.1.4) w.r.t $\boldsymbol{\alpha}$ and $\boldsymbol{\gamma}$ simultaneously and equate to zero

$\frac{d}{d \boldsymbol{\alpha}} E\left(e_{y} \boldsymbol{\alpha}^{\prime} \mathbf{e}_{r}-Y \boldsymbol{\gamma}^{\prime} \mathbf{X}_{s} \mathbf{e}_{s}\right)^{2}=0$,

or

$$
\begin{gathered}
-E\left(\mathbf{e}_{r} e_{y}\right)-E\left(\mathbf{e}_{r} \mathbf{e}_{r}^{\prime}\right) \boldsymbol{\alpha}+Y E\left(\mathbf{e}_{r} \mathbf{e}_{s}^{\prime}\right) \mathbf{X}_{s} \boldsymbol{\gamma}=0, \\
-\mathbf{S}_{y x_{r}}-\boldsymbol{\Sigma}_{x_{r}} \boldsymbol{\alpha}+Y \boldsymbol{\Sigma}_{x_{r} x_{s}} \mathbf{X}_{s} \boldsymbol{\gamma}=0, \\
-\mathbf{S}_{y x_{r}}=\boldsymbol{\Sigma}_{x_{r}} \boldsymbol{\alpha}-Y \boldsymbol{\Sigma}_{x_{r} x_{s}} \mathbf{X}_{s} \boldsymbol{\gamma} .
\end{gathered}
$$

where $\quad E\left(\mathbf{e}_{r} \mathbf{e}_{y}\right)=\left[\sum_{k=1}^{N} \Lambda_{k l} \hat{y}_{k} \hat{x}_{k i}\right]_{r \times 1}=\mathbf{S}_{y x_{r}}$,

$$
\begin{gathered}
E\left(\mathbf{e}_{r} \mathbf{e}_{r}^{\prime}\right)=\left[\sum_{k=1}^{N} \sum_{l=1}^{N} \Lambda_{k l} \hat{x}_{k i} \hat{x}_{l j}\right]_{r \times r}=\boldsymbol{\Sigma}_{x_{r}} \text { and } \\
E\left(\mathbf{e}_{r} \mathbf{e}_{s}^{\prime}\right)=\left[\sum_{k=1}^{N} \sum_{l=1}^{N} \Lambda_{k l} \hat{x}_{k i} \hat{x}_{l j}\right]_{r \times s}=\boldsymbol{\Sigma}_{x_{r} x_{s}} .
\end{gathered}
$$

Now by differentiating the equation (2.1.4) w.r.t $\boldsymbol{\gamma}$, we have

or

$$
\frac{d}{d \boldsymbol{\gamma}} E\left(e_{y}+\boldsymbol{\alpha}^{\prime} \mathbf{e}_{r}-Y \boldsymbol{\gamma}^{\prime} \mathbf{X}_{s} \mathbf{e}_{s}\right)^{2}=0
$$

$$
-E\left(\mathbf{e}_{s} e_{y}\right)-E\left(\mathbf{e}_{s} \mathbf{e}_{r}^{\prime}\right) \boldsymbol{\alpha}+Y E\left(\mathbf{e}_{s} \mathbf{e}_{s}^{\prime}\right) \mathbf{X}_{s} \boldsymbol{\gamma}=0,
$$$$
-\mathbf{S}_{y s}-\boldsymbol{\Sigma}_{x_{s} x_{r}} \boldsymbol{\alpha}+Y \boldsymbol{\Sigma}_{x_{s}} \mathbf{X}_{s} \boldsymbol{\gamma}=0 \text {, }
$$

or

$$
-\mathbf{S}_{y s}=\boldsymbol{\Sigma}_{x_{s} x_{r}} \boldsymbol{\alpha}+Y \boldsymbol{\Sigma}_{x_{s}} \mathbf{X}_{s} \boldsymbol{\gamma} .
$$

where

$$
E\left(\mathbf{e}_{s} \mathbf{e}_{y}\right)=\left[\sum_{k=1}^{N} \Lambda_{k l} \hat{y}_{k} \hat{x}_{k i}\right]_{s \times 1}=\mathbf{S}_{y x_{s}},
$$

$E\left(\mathbf{e}_{s} \mathbf{e}_{s}^{\prime}\right)=\left[\sum_{k=1}^{N} \sum_{l=1}^{N} \Lambda_{k l} \hat{x}_{k i} \hat{x}_{l j}\right]_{s \times s}=\Sigma_{x_{s}}$ and

$E\left(\mathbf{e}_{r} \mathbf{e}_{s}^{\prime}\right)=\left[\sum_{k=1}^{N} \sum_{l=1}^{N} \Lambda_{k l} \hat{x}_{k i} \hat{x}_{l j}\right]_{r \times s}=\boldsymbol{\Sigma}_{x_{r} x_{s}}$.

Now (2.1.5) and (2.1.6) can be written in matrix form as

$$
\left[\begin{array}{l}
\boldsymbol{\alpha} \\
\boldsymbol{\gamma}
\end{array}\right]=\left[\begin{array}{cc}
\boldsymbol{\Sigma}_{x_{r}} & -Y \boldsymbol{\Sigma}_{x_{r} x_{s}} \mathbf{X}_{s} \\
\boldsymbol{\Sigma}_{x_{s} x_{r}} & -Y \boldsymbol{\Sigma}_{x_{s}} \mathbf{X}_{s}
\end{array}\right]^{-1}\left[\begin{array}{l}
-\mathbf{S}_{y x_{r}} \\
-\mathbf{S}_{y x_{s}}
\end{array}\right] .
$$

The results for computing the inverse of matrix of matrices has been provided (Ahmad et al., 2014). According to their results we have

$$
\begin{gathered}
{\left[\begin{array}{c}
\boldsymbol{\alpha} \\
\boldsymbol{\gamma}
\end{array}\right]=\left[\begin{array}{cc}
\boldsymbol{\Sigma}_{x_{r}}^{-1}+Y \boldsymbol{\Sigma}_{x_{r}}^{-1} \boldsymbol{\Sigma}_{x_{r} x_{s}} \mathbf{X}_{s} \mathbf{H}_{s}^{-1} \boldsymbol{\Sigma}_{x_{s} x_{r}} \boldsymbol{\Sigma}_{x_{r}}^{-1} & -Y \boldsymbol{\Sigma}_{x_{r}}^{-1} \boldsymbol{\Sigma}_{x_{r} x_{s}} \mathbf{X}_{s} \mathbf{H}_{s}^{-1} \\
\mathbf{H}_{s}^{-1} \boldsymbol{\Sigma}_{x_{s} x_{r}} \boldsymbol{\Sigma}_{x_{r}}^{-1} & -\mathbf{H}_{s}^{-1}
\end{array}\right],\left[\begin{array}{l}
-\mathbf{S}_{y x_{r}} \\
-\mathbf{S}_{y x_{s}}
\end{array}\right]} \\
\text { where } \mathbf{H}_{s}^{-1}=\boldsymbol{Y}^{-1} \mathbf{W}_{s}^{-1}, \\
\mathbf{W}_{s}^{-1}=-Y^{-1}\left(\boldsymbol{\Sigma}_{x_{s}} \mathbf{X}_{s}-\boldsymbol{\Sigma}_{x_{s} x_{r}} \boldsymbol{\Sigma}_{x_{r}}^{-1} \boldsymbol{\Sigma}_{x_{r} x_{s}} \mathbf{X}_{s}\right)^{-1}
\end{gathered}
$$

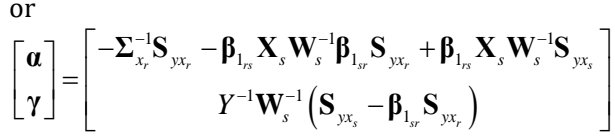

where $\boldsymbol{\beta}_{1_{r \mathrm{~s}}}=\boldsymbol{\Sigma}_{x_{r}}^{-1} \boldsymbol{\Sigma}_{x_{r} x_{\mathrm{s}}}$,

$\boldsymbol{\alpha}=-\boldsymbol{\Sigma}_{x_{r}}^{-1} \mathbf{S}_{y x_{r}}-\boldsymbol{\beta}_{1_{r s}} \mathbf{X}_{s} \mathbf{W}_{s}^{-1} \boldsymbol{\beta}_{1_{s r}} \mathbf{S}_{y x_{r}}+\boldsymbol{\beta}_{1_{r s}} \mathbf{X}_{s} \mathbf{W}_{s}^{-1} \mathbf{S}_{y x_{s}}$.

$$
\boldsymbol{\gamma}=Y^{-1} \mathbf{W}_{s}^{-1}\left(\mathbf{S}_{y x_{s}}-\boldsymbol{\beta}_{1_{s r}} \mathbf{S}_{y x_{r}}\right) .
$$

The MSE obtained by taking square and then expectation of equation (2.1.2). Also by substituting the value of $\boldsymbol{\alpha}^{\prime} s$ and $\gamma^{\prime} s$ from (2.1.7) and (2.1.8), the final expression of mean square error as $\operatorname{MSE}\left(t_{g m_{r c r}}\right)=s_{y_{s y s}}^{2}-\mathbf{S}_{y x_{r}}^{\prime} \boldsymbol{\Sigma}_{x_{r}}^{-1} \mathbf{S}_{y x_{r}}+\left(\mathbf{S}_{y x_{s}}^{\prime}-\mathbf{S}_{y x_{r}}^{\prime} \boldsymbol{\beta}_{1_{r s}}\right)$.

$$
\mathbf{W}_{s}^{-1} \mathbf{X}_{s}\left(\boldsymbol{\beta}_{1_{s r}} \mathbf{S}_{y x_{r}}-\mathbf{S}_{y x_{s}}\right)
$$

$$
\begin{aligned}
& \text { Where } E\left(\mathbf{e}_{y}^{2}\right)=\sum_{k=1}^{N} \Lambda_{k l} \hat{y}_{k i}^{2}=s_{y_{s y s}}^{2}, \\
& E\left(\mathbf{e}_{r} \mathbf{e}_{y}\right)=\left[\sum_{k=1}^{N} \Lambda_{k l} \hat{y}_{k} \hat{x}_{k i}\right]_{r \times 1}=\mathbf{S}_{y x_{r}} \\
& E\left(\mathbf{e}_{s} \mathbf{e}_{y}\right)=\left[\sum_{k=1}^{N} \Lambda_{k l} \hat{y}_{k} \hat{x}_{k i}\right]_{s \times 1}=\mathbf{S}_{y x_{s}} \text { and } \\
& E\left(\mathbf{e}_{r} \mathbf{e}_{r}^{\prime}\right)=\left[\sum_{k=1}^{N} \sum_{l=1}^{N} \Lambda_{k l} \hat{x}_{k i} \hat{x}_{l j}\right]_{r \times r}=\boldsymbol{\Sigma}_{x_{r}} . \\
& E\left(\mathbf{e}_{r} \mathbf{e}_{r}^{\prime}\right)=\left[\sum_{k=1}^{N} \sum_{l=1}^{N} \Lambda_{k l} \hat{x}_{k i} \hat{x}_{l j}\right]_{r \times r}=\boldsymbol{\Sigma}_{x_{r}} .
\end{aligned}
$$

\subsection{Jackknife Replication Variance}

The Jackknife variance estimator for the estimator developed in (2.1.1) is considered and the Jackknife replicates for (2.1.1) after successively deleting $k^{\text {th }}$ units are: $t_{s m_{m a}}^{(k)}=\left[t_{y}^{t_{y}^{(k)}}+a \sum_{i=1}^{r} \alpha_{i}\left(t_{x_{i}}^{f^{(k)}}-X_{i}\right)\right]\left[\prod_{i=r+1}^{s}\left(\frac{X_{i}}{t_{x_{i}}^{(k)}}\right)^{\alpha_{i}}\right] \cdot \quad$ (2.2.1) Where $t_{y}^{\prime(k)}= \begin{cases}\sum_{s_{k}} \frac{y_{k}}{\pi_{k}} & \text { if } \quad k \in s_{k} \\ \sum_{s} \frac{y_{k}}{\pi_{k}} & \text { if } \quad k \in S\end{cases}$ (2.2.2)

and

$t_{x_{i}}^{(k)}= \begin{cases}\sum_{s_{k}} \frac{x_{k}}{\pi_{k}} & \text { if } \quad k \in s_{k} \\ \sum_{s} \frac{x_{k}}{\pi_{k}} & \text { if } \quad k \in S\end{cases}$

where the sample with $k^{\text {th }}$ units being deleted belongs to $S_{k}$ and complete sample belongs to $s$.

The pseudo-value defined as

$$
t_{j_{r c r}}=n t_{g m_{r c r}}-(n-1) t_{g m_{r c r}}^{(k)},
$$

where $t_{g m_{r e r}}$ is defined in (2.1.1) and $t_{g m_{r c r}}^{(k)}$ defined in (2.2.1).

The Jackknife variance estimator is

$$
v\left(t_{j_{r c r}}\right)=\frac{1}{n(n-1)} \sum_{i=1}^{n}\left(t_{j_{r c r}}-t_{J K_{r e r}}\right)^{2},
$$

where $t_{J K_{r c r}}$ defined as 
$t_{J K_{r c r}}=\frac{1}{n} \sum_{i=1}^{n} t_{j_{r c r}}$

The Jackknife variance estimator form as

$$
\begin{aligned}
& v\left(t_{j_{r c r}}\right)=\frac{1}{n(n-1)} \sum_{i=1}^{n}\left(t_{j_{r c r}}-t_{J K_{r e r}}\right)^{2}, \\
& =\frac{1}{n(n-1)} \sum_{i=1}^{n}\left(\left(n t_{g m_{r r}}-(n-1) t_{g m_{r r}}^{(k)}\right)-\frac{1}{n} \sum_{i=1}^{n}\left(n t_{g m_{r r}}-(n-1) t_{g m_{r e r}}^{(k)}\right)\right)^{2},
\end{aligned}
$$$$
=\frac{(n-1)^{2}}{n(n-1)} \sum_{i=1}^{n}\left(t_{g m_{r c r}}^{(k)}-\frac{1}{n} \sum_{i=1}^{n} t_{g m_{r c r}}^{(k)}\right)^{2} \text {, }
$$

Or

$$
\begin{aligned}
& v\left(t_{j_{k r}}\right)=\frac{n-1}{n} \sum_{i=1}^{n}\left(\left[t_{y}^{\prime(k)}+a \sum_{i=1}^{r} \alpha_{i}\left(t_{x_{i}}^{\prime(k)}-X_{i}\right)\right]\left[\prod_{i=r+1}^{s}\left(X_{i} / t_{x_{i}}^{(k)}\right)^{c_{j}}\right]\right. \\
& \left.-\frac{1}{n} \sum_{i=1}^{n}\left(\left[t_{y}^{(k)}+a \sum_{i=1}^{r} \alpha_{i}\left(t_{x_{i}}^{(k)}-X_{i}\right)\right]\left[\prod_{i=r+1}^{s}\left(X_{i} / t_{x_{i}}^{\prime(k)}\right)^{c \gamma_{i}}\right]\right)\right)^{2},
\end{aligned}
$$

or

$$
\begin{aligned}
& v\left(t_{j_{k r}}\right)=\frac{n-1}{n} \sum_{s}\left(\left[\sum_{s_{k}} y_{k} / \pi_{k}+a \sum_{i=1}^{r} \alpha_{i}\left(\sum_{s_{k}} x_{k i} / \pi_{k}-X_{i}\right)\right]\left[\prod_{i=r+1}^{s}\left(X_{i} / \sum_{s_{k}} x_{k i} / \pi_{k}\right)^{c_{k}}\right]\right. \\
& \left.-\frac{1}{n} \sum_{s}\left(\left[\sum_{s_{k}} y_{k} / \pi_{k}+a \sum_{i=1}^{r} \alpha_{i}\left(\sum_{s_{k}} x_{k i} / \pi_{k}-X_{i}\right)\right]\left[\prod_{i=r+1}^{s}\left(X_{i} / \sum_{s_{k}} x_{k i} / \pi_{k}\right)^{c_{i}}\right]\right)\right)^{2} .
\end{aligned}
$$

Different special cases of proposed class have also been developed under systematic sampling with varying probabilities. The expressions of Bias, mean square and their Jackknife replication variance estimators for the special class of estimators as follows;

\subsection{Special Cases}

The performance of regression estimator is similar to ratio estimator when relationship passing through origin. But in many practical situations when regression line not passed through the neighbourhood of origin in this case

\begin{tabular}{|c|c|c|}
\hline Special cases & $\begin{array}{l}\text { Special Case-I(Regression Estimator) } \\
\text { when } a=1, \quad c=0 \text { substitute in 2.1.1 }\end{array}$ & $\begin{array}{l}\text { Special Case-II (Ratio Estimator) } \\
\text { When } c=1 \text { and } a=0 \text { substitute in } 2.1 .1\end{array}$ \\
\hline \begin{tabular}{|l|} 
Estimator \\
\end{tabular} & 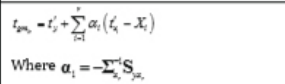 & 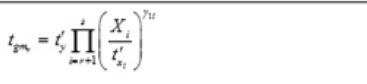 \\
\hline Bias & $\operatorname{Bias}\left(t_{t m_{s}}\right)=0$ & 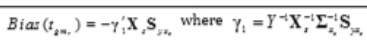 \\
\hline Mean Square Error & 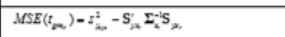 & 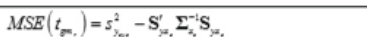 \\
\hline \begin{tabular}{|l}
$\begin{array}{l}\text { Replication Variance } \\
\text { estimator }\end{array}$ \\
\end{tabular} & 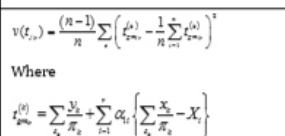 & 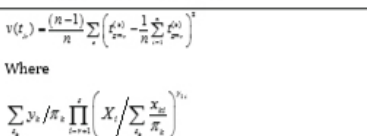 \\
\hline
\end{tabular}
regression performs better than ratio estimator (Singh and Espejo, 2003). The number of special cases of proposed class given in (2.1.1) with their expression of bias, mean square errors and Jackknife replication variance estimators are given in the followingtable;

\section{Table 2.1 Special Cases under Systematic Sampling}

\section{Simulation Study}

In this section conducted a simulation study, A finite artificial population of size $N=1000$ with six auxiliary variables $\left(x_{1}, x_{2}, \ldots, x_{6}\right)$, where the population elements are independently generated from $x_{1} \square N(2.1,0.79), x_{2} \square N(7,1.2), x_{3} \square N(11,1.8)$, $x_{4} \square N(9,2.3), x_{5} \square N(6,4.5)$, and $x_{6} \square N(12,2.7)$. The model is

$y_{i}=\beta_{0}+\beta_{1} x_{1}+\beta_{2} x_{2}+\ldots . .+\beta_{6} x_{6}+e_{i}$ with

$\left(\beta_{0}, \beta_{1}, \beta_{2}, \ldots, \beta_{6}\right)=(0.23,3,2.3,2.20,1.75,1.30,5) \quad$ and

$e_{i} \square N(0,1)$. The variable $z_{i} \square N(8,3.5)$ was used as a size measure for the unequal probability sampling.

The results generated for 4,000 samples where a sample of $n=200$ is selected by employing the scheme of sampling, when the order of the population units is random (Madow, 1949). This systematic selection procedure is given in R language software and by default its command is $s=$ UPrandomsystematic(pik,eps=1e-6) where pik is a vector of inclusion probability and eps control value which is by default equal to $1 \mathrm{e}-6$. Inclusion probabilities calculated by $\pi_{k}=\frac{n X_{k}}{X}$, it can also get by default command in $\mathrm{R}$ language, i.e. pik=inclusionprobabilities(a,n) where "a "is vector of positive integer and $\mathrm{n}$ size of sample.

Firstly, for replication variance estimation, we estimate parameter from the whole sample $n=200$ then dropping one unit of sample and then recalculated the statistic of interest based on the incomplete sample $n-1=199$. A pseudo-value is then computed as the difference between the whole sample estimate and the partial estimate from incomplete sample. Using these pseudo values we calculated estimated total, Jackknife replication variance estimate and also $95 \%$ confidence interval.

\subsection{Analysis of Proposed Estimators}

For each of the $S=4000$ samples, percent relative bias of the total estimator and the Jackknife variance estimates is calculated with respect to the parameter of interest (population total $T$ ) and true mean squared error respectively i.e.,

$$
\text { P.R.B }=\frac{E(t)-T}{T} \times 100,
$$

where

$$
\begin{gathered}
E(t)=\frac{1}{4000} \sum_{i=1}^{4000} t_{i} . \\
P . R . B=\frac{E\left(V_{J}(t)\right)-M S E_{\text {true }}}{M S E_{\text {true }}} \times 100^{\prime}
\end{gathered}
$$

where

$$
E\left(V_{J}(t)\right)=\frac{1}{4000} \sum_{i=1}^{4000} V_{J}\left(t_{i}\right) \text { and } \quad M S E_{\text {true }}=\frac{1}{4000} \sum_{i=1}^{4000}\left(t_{i}-T\right)^{2} .
$$

The 95 percent confidence interval coverage by each of the variance estimators for the proposed estimators is:

$$
\text { mean }(\text { pseudo }) \pm t_{1-\alpha / 2, n-1} \sqrt{\operatorname{var}(\text { pseudo }) / n}
$$

\subsection{Simulation Results}

The results for 4,000 samples for total estimators with their bias, mean square error, replication variance, percent relative bias and confidence intervals are present in Table1, 2 and 3.

Table 1: Bias, MSE \& Replication Variance Estimates for $(4,000$ Samples) 


\begin{tabular}{|c|c|c|c|}
\hline Estimator & Bias & $\begin{array}{l}\text { Mean Square } \\
\text { Error }\end{array}$ & $\begin{array}{c}\text { Replication } \\
\text { Variance Estimates }\end{array}$ \\
\hline$t_{g_{m_{l r}}}$ & -89.10782 & 40136582 & 43247768 \\
\hline$t_{g_{m_{r}}}$ & -38.04006 & 8613089 & 482425.88 \\
\hline$t_{g_{m_{r c r}}}$ & 13.02923 & 4198198 & 70335115 \\
\hline
\end{tabular}

In this study, we have developed a general class of estimators for estimating the population total using multi-auxiliary variables under systematic PPS sampling design with varying probabilities. The overall result showed that the variances are calculated by Jackknife variance estimators with smaller bias and confidence intervals using these replication estimates provides precise interval. We may also conclude from simulation regarding performance of proposed estimators, the class of regression-cum-ratio has smaller MSE with least bias than all suggested estimators so we preferred this estimators.

Table-1 represents the results of bias, mean square error and replication variance for the point estimators, based on systematic sampling design with varying probabilities are regression, ratio and regression-cumratio estimator. According to table- 1 there are regression-cum-ratio estimator which have minimum MSE with smaller but positive bias about (13.0292) than other estimators. The regression and ratio have high MSE but negative bias. From table-1 we can see that the variance estimator using Jackknife replication variance is smaller for ratio while regressioncum-ratio estimator has greater replication variance.

Table 2: Relative bias (RB) of Total for the proposed estimators $(4,000$ samples)

\begin{tabular}{ccc} 
Estimator & Estimated Total & RB (\%) \\
\hline$t_{g_{m_{l r}}}$ & 1480.608 & -0.9868941 \\
$t_{g_{m_{r}}}$ & 1853.468 & -0.9896118 \\
$t_{g_{m_{r c r}}}$ & 1887.988 & -0.9880399 \\
\hline
\end{tabular}

Table- 2 shows the percent relative biases (PRB) of the eight types of estimators for total. All the biases are less than $1 \%$ in the absolute value and according to findings of relative biases all these estimators behave reasonably in term of point estimation. Table- 3 represents the percent relative bias (PRB) of Jackknife variance estimator and confidence interval (C-I) for the proposed estimators. The results show that, regression estimator has smaller positive relative bias as compared to other estimators. On the other hand ratio having negative PRB.

Table 3: Relative bias (RB) of Jackknife Variance Estimator and Confidence Interval (C-I) for the Proposed estimators $(4,000$

\begin{tabular}{ccccc} 
Estimator & $\begin{array}{c}\text { Estimated } \\
\text { Total }\end{array}$ & S.E & Ramples) & $\begin{array}{c}\mathbf{9 5 \%} \\
\text { Confidence Interval }\end{array}$ \\
\hline$t_{g_{m_{l r}}}$ & 1480.608 & 465.14 & 0.0775 & $563.40-2397.82$ \\
$t_{g_{m_{r}}}$ & 1853.468 & 49.11 & -0.9439 & $1756.63-1950.31$ \\
$t_{g_{m_{r c r}}}$ & 1887.988 & 593.02 & 15.7536 & $718.62-3057.10$ \\
\hline
\end{tabular}

Table- 3 represents the confidence interval for population total using standard error which generated through Jackknife variance estimator. For this purpose generate 4000 samples of size 200 and for each sample, we compute the Jackknife 95\% confidence interval. According to those results, we are $95 \%$ confident to say that this interval will contain the true value of population total. It is important to note that the narrow intervals of estimators leads to a higher precision than wider intervals. The ratio estimator have narrow limits to each other than others.

\section{Conclusion}




\section{References}

[1] Ahmad, Z., Afzal, M., Shahid, U. and Hanif, M. (2014). A General Class of Mean Estimators Using Mixture of Auxiliary Variables for Two-Phase Sampling in the Presence of Non-Response at Second Phase for No Information Case. Pakistan Journal of Statistics., 30(3): 291-318.

[2] Des R., (1964). Systematic Sampling with PPS in a Large Scale Survey. American Statistical Association.59(305), 251-255.

[3] European Communities (2002). Monographs of Official Statistics Variance Estimation Methods in the European Union. Research in official statistics.31-43.

[4] Goga, C. (2008). Variance Estimators in Survey Sampling. Universite de Bourgogne Dijon, France.

[5] Gregoire, T. G., Li, Z., (2011). An Emprical Evaluation of the Accuracy of the Hartley and Rao (1962) Variance Approximation Following PPS Systematic Sampling. Pakistan Journal of Statistics, 27(4):555-566.

[6] Hartley, H.O., (1966). Systematic Sampling with Unequal Probability and Without Replacement. Journal of American Statistical Association, 61(315):739-748.

[7] Hartley, H.O., Rao, J.N.K., (1962). Sampling with Unequal Probabilities and Without Replacement. The Annals of Mathematical Statistics, 33 (2): 350-374.

[8] Horvitz, D. G., Thompson, D. J., (1952). A Generalization of Sampling Without Replacement from a Finite Universe. Journal of the American Statistical Association, 47(260): 663-685.

[9] Madow, W.G., (1949). On the Theory of Systematic Sampling II. Annals of Mathematical Statistics, 20 (3):333-354.

[10] Madow, W.G., Madow, L.H., (1944). On the Theory of Systematic Sampling I. Annals of Mathematical Statistics, 15(1):1-24.

[11] Midzuno, H., (1950). An Outline of the Theory of Sampling System. Annals of the Institute of Mathematical Statistics, 1(2):149-156.

[12] Murthy, M. N., (1967). Sampling Theory and Methods. Statistical Publishing Society, Calcutta.

[13] Rao, J. N. K., Shao, J., (1992). Jackknife Variance Estimation with Survey Data under Hot Deck Imputation. Biometrika, 79(4): 811-822.

[14] Roy, D., Safiquzzaman, M. (2000). Jackknifing a General Class of Estimators. Brazilian Journal of Probability and Statistics, 14 (1): 47-60.

[15] Roy, D., Safiquzzaman, M. (2002).Variance Estimation by Jackknifing Method: A New Approach with Reference to Varying Probability Sampling. Calcutta Statistical Association Bulletin Proceedings, 52 (1-4): 205-348.

[16] Roy, D., Safiquzzaman, M., (2006). Variance Estimation by Jackknife Method under Two-Phase Complex Survey Design. Journal of Official Statistics, 22(1), 35-51.

[17] Yates, F., Grundy, P.M., (1953). Selection without Replacement from within Strata with Probability Proportional to Size. Journal of Royal Statistics Society Series, 15(2): 153-161. 